Horizons philosophiques

\title{
Le statut sémiotique de la perspective dans l'oeuvre picturale
}

\section{Marie Carani}

Volume 1, numéro 2, printemps 1991

Sémiotiques 2 : théories et champs d'application

URI : https://id.erudit.org/iderudit/800870ar

DOI : https://doi.org/10.7202/800870ar

Aller au sommaire du numéro

\section{Éditeur(s)}

Collège Édouard-Montpetit

\section{ISSN}

1181-9227 (imprimé)

1920-2954 (numérique)

Découvrir la revue

Citer cet article

Carani, M. (1991). Le statut sémiotique de la perspective dans l'oeuvre

picturale. Horizons philosophiques, 1(2), 13-31. https://doi.org/10.7202/800870ar d'utilisation que vous pouvez consulter en ligne.

https://apropos.erudit.org/fr/usagers/politique-dutilisation/ 


\section{Le statut sémiotique de la perspective dans l'œuvre picturale}

\section{Problématique}

En arts visuels, la sémiotique est née historiquement du discours verbal, elle s'est développée par rapport à la théorie linguistique comme une méthode qui voulait comprendre le fonctionnement de l'image fixe (peinture, photographie) ou en mouvement (cinéma, vidéo). Sur cette base, les sémioticiens de l'image ont choisi d'appliquer les systèmes de signes de la langue à la compréhension et à l'interprétation du référent, ce qui suggérait et définissait d'entrée de jeu un contenu. En peinture figurative ou abstraite, notamment, cette question du référent devenait ce que l'artiste et le regardeur étaient capables ou se permettaient de nommer. Le sens d'une image concernait ainsi a fortiori les choses reconnaissables, c'est-à-dire ressemblantes par "degrés d'iconicité", dans la foulée des thèses icono-sémiologiques de Barthes et Marin, ou des conceptions positivistes américaines depuis Peirce.

Après Saussure, les développements de la linguistique verbale comme science des signes verbaux et non verbaux avaient pourtant permis d'envisager qu'une théorie du langage visuel était possible sur la base d'un véritable méta-langage qui rendrait compte de la dimensionnalité particulière de ce discours. De même, à l'instar de Peirce, 
plusieurs analystes de l'image peinte l'avaient caractérisée d'hypoicône en raison de sa "saveur propre, immédiate" qui dépasserait les systèmes de catégories habituels (icône/indice/symbole) développés pour en rendre compte1. Mais ces expériences de l'espace se sont montrées, à l'usage, exclusivement préoccupées de décrire les paramètres de la notion d'objet autonome, liée intrinsèquement à la pensée conceptuelle et formelle (vs spatiale). Simples prolongements de l'iconologie, ces discours sémiotiques n'ont pas été capables de décrire la structure du champ spatial au-delà de ce qui est perçu et reconnu comme des unités (entités) iconographiques. On aura préféré la dénotation imagière à l'en deçà de la figure. C'est dire que la raison sémiotique elle-même s'est plus ou moins empêtrée dans ce leurre, qu'elle est arrivée avec peine à s'en soustraire, l'approche privilégiée par la discipline ayant été caractérisée par le souci de décrire la forme per se de l'objet observé, en l'occurrence des éléments de langage iconique.

Quand l'historien d'art Meyer Schapiro s'est intéressé en 1969 au champ de l'image iconique ${ }^{2}$, c'est-à-dire aux véhicules-signes non mimétiques de la surface peinte : cadre, contours des formes, frontières des plans, périphéries, contrastes, il aura été l'un des premiers théoriciens à concevoir que les fondements de la sémiotique visuelle pouvaient reposer sur autre chose qu'une notion philosophico-esthétique de mimesis qui remonterait à Platon.

1. Voir Charles Peirce, Écrits sur le signe, traduction de Gérard Deledalle, Seuil, Paris, 1978, p. 120; Hubert Damisch, uHuit thèses pour (ou contre?) une sémiologie de la peinture", Macula, 2, 1977, p. 17-23; "Sémiologie et iconographie", La sociologie de l'art et sa vocation interdisciplinaire, Gabienne Francastel éd., Denoel/Gonthier, Paris, 1976, p. 29-39.

2. Meyer Schapiro, "Sur quelques problèmes de sémiotique de l'art visuel : champ et véhicule dans les signes iconiques", in Critique, 315-316, aoûtseptembre 1973, p. 843-866. 
Transgressant le credo de la rationalité verbale, le nommé, qui présiderait à la clarté du tableau, Schapiro propose déjà la présence d'un infra-texte iconique, préalable à tout investissement imagier, dont il vaudrait la peine d'aborder la fonction dans le système de la représentation comme modèle syntaxique.

Depuis, en vue de constituer une théorie générale de la communication visuelle, Umberto Eco a démontré qu'en plus du langage iconique, il existe toujours dans le champ visuel des configurations matérielles (gestaltistes, graphiques/perspectivistes) qui stimulent et sous-tendent les conditions de perception d'objets ou d'événements dans la multiplicité du réel ${ }^{3}$. Eco pose que, pour décrire la composante majeure du contenu imagier, c'est-à-dire l'iconisme et ses effets, il est impossible de ne pas reconnaître comme soubassement, le principe d'une hypothèse spatiale, d'un régulateur perspectif au niveau des distances ou des intervalles instaurés entre les éléments visuels et plastiques constitutifs. Au même titre que l'iconicité fait irruption à la surface de l'œuvre par le biais de motifs iconiques, la systématicité perspective s'inscrirait dans les profondeurs du tableau par sa mise en œuvre de moyens ou d'outils plastiques : structure linéaire, vectorialités, relations point de vue/point de fuite, chiaroscuro, raccourcis, etc., moyens qui prennent résolument en charge l'unité picturale.

Eco définit ainsi une surface peinte ancrée dans une structure visuelle que l'on peut appeler texte, qui est une sub-structure sémiotique autre que la procédure de transposition d'un système verbal (le discours iconique) dans un autre (le visible). Contrant l'arrêt sur l'iconicité, Eco pointe comme objet théorique immédiat une notion de système du tableau qui n'est pas à proprement parler idiolectale, mais spatialisante, perspectiviste, qui, toujours là, joue 
en dessous du dire. Dans le même sens, dans son récent ouvrage L'origine de la perspective ${ }^{4}$, Hubert Damisch souligne que ce paradigme de la représentation visuelle aura constitué une première modélisation de l'énonciation visuelle permettant "de penser, en peinture, et dans les formes, par les moyens, du point de vue qui est le sien» 5 .

Damisch rappelle que la perspective fournit à la peinture, depuis la Renaissance italienne, un appareil formel qui se rapproche de celui de l'énonciation en linguistique, avec lequel il présente de nombreux traits communs, notamment deux composantes déterminantes : 1) la distribution que cet appareil organise des points de vue, points de fuite et de distance, c'est-à-dire ce que Damisch nomme l'ici, le là et le là-bas, véritable géométrie de l'énonciation ayant son analogue au registre figuratif; 2) la place que cet appareil de base assigne au sujet dans le réseau dramatique-narratif déjà constitué, et qui confère un sens à sa visée, en même temps qu'il ouvre la possibilité d'un "énoncée en peinture. Damisch reconnaît ainsi, dans le processus même de la mise en perspective, une proposition englobante dotée d'un sens, d'une direction.

Damisch précise pourtant que, malgré ces facultés énonciatrices qui l'accomplissent, si la perspective tend au discours comme à sa fin ou sa raison d'être, elle a par contre son origine (ou son départ) ailleurs que dans l'élément de la parole. Elle s'ancre au contraire, nous dit-il, dans le plan "où s'inscrit la peinture, là où elle travaille et se réfléchit et où la perspective la démontre"6. L'aspect peu satisfaisant et inadéquat d'un mode de translation linguistique du phénomène perspectiviste est ainsi reconnu par Damisch, qui suggère comme Eco l'existence d'un

4. Hubert Damisch, L'origine de la perspective, Flammarion, Paris, 1987.

5. Ibid., p. 405.

6. Ibid., p. 407. 
processus pré- ou sub-discursif autre que la formulation iconique, pour donner à voir, à lire et à comprendre le contenu de la représentation visuelle. II n'aborde toutefois pas la mise en place de cette structure profonde du tableau qu'il associe à sa qualité spatiale intrinsèque. II laisse à d'autres le soin d'en démont(r)er l'infrastructure, suggérant cependant que le décodage d'un langage spatial procéderait comme suit : au lieu d'établir des parcours du regard qui seraient éduqués par ce qui est reconnaissable, on voudrait d'abord éclairer la structure même du champ visuel. Une telle entreprise permettrait de dénoncer l'idéalisme anti-matérialiste d'un mode purement figural de représentation.

En ce sens, il m'apparaît opportun d'aborder, puis d'explorer l'épaisseur de la peinture, si l'on veut, l'instance graphique qui permet de donner le pouvoir de représentativité, le niveau d'organisation et d'association qui va aider la perception à reconnaître. Là, on creuse sous les codes iconiques, mais on ne les élimine pas; on ne se limite pas à des régions-formes susceptibles de recevoir une nomenclature verbale. On interroge plutôt structuralement le réseau d'éléments qui se constitue en un plan du contenu, significatif par lui-même, mais situé dans une position syntaxique intermédiaire de passage, de frontière entre le plan de l'expression, celui des variables constitutives ou signifiants premiers du tableau (variation des éléments formels, type de touche, textures, tonalités, dimension des formes, vectorialités, chromatisme) révélés par la sémiologie topologique ${ }^{7}$, et le niveau du sémantique, du sens signifié.

Dans ce contexte, si on considère la figuration comme critère de vérité, de vraisemblance, même la peinture abstraite du 20 e siècle, tout en réalisant la plus importante révolution dans le domaine de l'iconique, n'a cessé d'im-

7. Fernande Saint-Martin, Sémiologie du langage visuel, Presses de l'Université du Québec, Sillery, 1987. 
pliquer des commentaires sur le sujet de la peinture allant d'une copie imparfaite des référents jusqu'à l'aniconicité pure, via la présence d'agrégats graphiques peu ou pas visibles de prime abord, mais dont l'association rend possible l'édification du sens en fonction de certaines boniformisations gestaltistes, graphémiques ou graphiques relevant de la pensée visuelle qui les produit et les manipule.

\begin{tabular}{|lll|}
\hline Structures profondes & & Surface \\
Syntaxique & (Passage de frontière) & Sémantique \\
Signifiants & $\begin{array}{l}\text { Contenu (sujet) } \\
\text { premiers de }\end{array}$ & Signifiés, \\
l'Expression & perspectif & sens signifié \\
& & \\
\hline
\end{tabular}

On retiendra que je veux examiner ici une structuration du contenu imagier qui ne dépend pas seulement de l'effet de réalité. L'iconicité n'est pas le seul modèle, ou le modèle le plus adéquat pour étudier le langage visuel, car sa description s'inscrit comme traduction purement linguistique de l'image peinte, d'où la limite de cette modélisation : celle de "dérivée» par rapport au pictural. En ne réduisant pas mon analyse à cette lecture incomplète, mais en considérant comme mon objet les composantes morphologiques de l'image-plan en tant que faits de langage, effets de structures, je postule l'existence d'une grammaire visuelle où le schéma structural, comme ses enjeux et sa définition même, n'est plus exclusivement basé sur le référent. II faut passer outre aux figures de la surface qui auront orienté trop longtemps le décodage sémiotique du tableau, il faut libérer la peinture, la délivrer du verbe, pour ressourcer la description et l'analyse même de l'image. 


\section{Passages disciplinaires}

Tout art est une pratique spatiale. Sémiotiser la production artistique comprend ainsi la question de la représentation dans le modèle. La représentation s'entend d'un ensemble de règles d'expression et d'opérationnalisation; chaque tableau est donc la mise en représentation d'un langage. Dans ce contexte, modéliser est toujours une procédure de méta-langage, un plan autre que celui de la manifestation visuelle concrète, et le langage visuel comme tel se veut mise en scène, se décrit, investit l'œuvre par inscription de variables visuelles et plastiques. Là, la sémiotique se présente comme le lieu d'élaboration d'une systématicité des signes visuels, et la grammaire visuelle, comme l'ensemble des procédures selon lesquelles des prégnances imagières sont converties en représentations sémantiques. Dès lors, toute esthétique est envisageable à partir d'hypothèses sur le mode de production des signifiants/signifiés visuels. En conséquence, je suggérerai que le langage visuel supporte une modélisation méta-linguistique à dimensions spatiales, qui s'offre comme une combinatoire de lignes, de plans, de positions et d'orientations des formes iconisées.

Cette orientation de recherche veut que l'univers visible soit ordonné en strata sur la base de la relation entre deux instances sémiotisables : l'iconicité et la perspectivité, tout en reconnaissant qu'il existe antérieurement (sémiotiquement parlant) des composants ou matériaux a-sémantiques de l'Expression, qu'il convient dans une analyse précédente (déjà réalisée par la sémiologie topologique) de reconnaître, d'isoler ou de classifier. De ces composants minimaux aux ensembles de signes nécessaires pour élaborer une totalité significative, comme un texte visuel, le sémioticien s'inscrit dans l'optique d'une tradition postsaussurienne : soit celle distinguant, depuis Benvéniste, sémiotique et sémantique, respectivement comme mode de description et d'identification syntaxiques, et comme 
mode discursif d'interprétation des référents repérés. Je me limiterai ici au plan proprement sémiotique, à ce premier lieu de passage de la matière vers le contenu imagier, préalable à la production comme telle du sens.

En général, les théories artistiques et celles de l'histoire de l'art ont expliqué le comment et le pourquoi de l'image à l'aide de deux grandes catégories dominantes: la forme et le contenu ${ }^{8}$. À cet égard, il semble que l'histoire ou la critique d'art et la sémiotique française ont en commun d'utiliser des notions analytiques assez similaires, essentiellement binaires. Pourtant la rigueur exercée en sémiotique dans l'utilisation des termes contraste avec son usage imprécis et approximatif dans les propositions esthético-stylistiques des historiens d'art. Pour substituer à des notions de forme et contenu apparents issues de l'évidence première, d'une conception basée sur la similarité mimétique, pour penser au-delà de l'héritage formel de l'histoire de l'art, le sémioticien visuel a convié les spécialistes de la production artistique à reconnaître dans ses efforts de modélisation de la matière visuelle les éléments fondamentaux du langage artistique. À la projection intuitive et sensible, aux hypothèses impressionnistes, la sémiotique oppose une certaine scientificité critique. Par exemple, même si l'histoire de l'art sait habituellement lire, faire sens d'un tableau par des contours (des figures) ou des rendus graphiques à haut degré d'iconicité, ce qui aura constitué grosso modo le contenu ou le sujet du tableau, l'historien d'art (au contraire du sémioticien) aura toujours occulté les organisations formelles abstraites, les réseaux de relations instaurées entre les unités de ce contenu.

Dit autrement, pour la sémiotique binariste qui sera ici la mienne, il est interdit de proposer une construction

8. Hans Belting, L'histoire de l'art est-elle finie?, éd. Jacqueline Chambon, Nîmes, 1989, p. 26-48. 
formelle de l'œuvre finie qui serait convoquée, comme en histoire de l'art, en référence exclusive à la discipline de l'iconologie, sans évoquer, pour comprendre, la rencontre et l'enchaînement hiérarchique du texte visuel. L'expérience de la double distinction hjelmslévienne entre expression et contenu, forme et substance ${ }^{9}$, m'amènera plutôt à opérer au niveau où s'élaborent les structures profondes, niveau qui permet de rendre compte de la façon dont la signification des formes et des contenus est engendrée structuralement. Partant, l'efficace du métier de sémioticien reposerait précisément sur la procédure de conversion d'une discipline à l'autre, et, comme l'a précisé Petitot ${ }^{10}$, la conversion pourrait constituer la charnière interdéfinitionnelle des concepts descriptifs et analytiques.

$\mathrm{Ma}$ (re)composition sémiotique du phénomène artistique sera ainsi dirigée à partir, et autour, du dépassement de la notion de "style», si largement utilisée en histoire de l'art sans qu'on en ait abordé véritablement la définition. Puisque celle-ci reste ouverte, voire insaisissable, on voit mal comment ce terme a pu devenir un instrument heuristique de connaissance. Parler du style d'un tableau, ce n'est ainsi qu'un piège, ce n'est pas le décrire, c'est plutôt l'inscrire dans des limites utiles, historiographiques, c'est lui assigner des bornes normatives, historiques et esthétiques, qui ont pour origine la rhétorique de l'écart.

Étant donné cependant l'extension sémantique attribuée à la notion de "description stylistique» en art, qui s'est imposée comme une nécessité de reconnaissance, tel l'inhérent du dire, la description semble s'énoncer, au plan méthodologique, comme le premier lieu d'inscription d'un transfert conceptuel de l'histoire de l'art vers la

9. Louis Hjelmslev, Prolégomènes à une théorie du langage, Minuit, Paris, 1968. 10. Jean Petitot, Morphogénèse du sens, PUF, Paris, 1985, p. 23-29. 
sémiotique. Comment envisager et éventuellement justifier ce pouvoir? Ne peut-on identifier une telle démarche dans la théorie même de l'histoire de l'art? Un retour sur certaines thèses de l'historien d'art Erwin Panofsky permet de démontrer la pertinence opératoire et les débouchés d'un faire-voir dédoublant conceptuellement un faire-sens.

\section{Du descripteur panofskien}

Selon Panofsky, le travail de la description présume déjà d'une interprétation des formes et des contenus présentés sur la toile, elle présuppose des principes généraux qui commandent (ou orientent) la représentation du tableau. II importerait donc pour l'analyste de considérer la verbalisation sous l'angle de sa structure sous-jacente ${ }^{11}$.

Panofsky anticipe au plan du tableau allégorique la question de la description comme méta-langage. Développant ses divisions sémantiques initiales (histoire des formes, histoire des types, histoire des idées), l'iconologue dégage de cet agrégat des "moments» qui interviennent relativement tardivement ou non dans le processus par lequel le spectateur reçoit l'œuvre, par lequel le regardeur jouxte chaque forme avec la chose-objet qu'elle représente. Panofsky tente d'indiquer que les processus perceptifs liés à tel ou tel type imagier de représentation, notamment leur mémorisation, sont mobilisés en vertu d'habitus culturels, c'est-à-dire des règles et des savoirs perspectifs qui étayent l'identification d'entités nommables et décelables à l'intérieur du champ visuel. Pour ce faire, il assimile les instances temporelles et physiques de l'interprétation à des niveaux de sens spécifiques contrôlant le déchiffrage tout au long de la description des

11. Erwin Panofsky, “Contribution au problème de la description d'œuvres appartenant aux arts plastiques et à celui de leur contenun, in La perspective comme forme symbolique, Minuit, Paris, 1976, p. 235-255. 
œuvres d'art. Encore en 1957, en tête de son ouvrage Meaning in the Visual Arts ${ }^{12}$, il restera fidèle à cette partition de 1932 publiée initialement dans La perspective comme forme symbolique.

Panofsky prescrit, par processus d'implication verticale, la présence d'un sens-chose dont le fonctionnement opère avec les formes enregistrées par la perception et les notions associées par l'expérience. C'est dire que le travail descriptif postule un mouvement quasi automatique de reconnaissance qui résulte de l'intériorisation non réfléchie de codes ou schèmes perspectifs préalables tant culturels qu'esthétiques. Le spectateur dégage du magma visuel de la matière plastique, l'image d'objets ou de personnages juxtaposés à des choses. Le caractère figural, la qualité d'image d'une peinture dépend ainsi d'un réseau complexe liant des séries de signes graphiques et des séries de figures. II ne reste plus alors au descripteur panofskien qu'à nommer les termes de ce méta-classement, qu'à isoler les opérations qui sont un texte de classement organisant une plasticité.

En outre, dans la transition du magma informé à l'objet-chose, à la forme - la chose réelle donnant son nom à la chose peinte - Panofsky reconnaît la pérennité d'un schéma structural qui chemine, par le jeu des classificateurs et des modificateurs, de l'englobant perméable à l'englobé, donc des profondeurs de l'image à la surface, rapport qui se reproduit systématiquement comme mode d'emboîtement du texte artistique. II en conclut que la couche première, largement pré-consciente, porte en elle les prémisses de la description, donc que ce sens-phénomène de base dans lequel se manifeste le tableau peut être considéré comme la cellule de la description à venir. II ajoute qu'ensuite, de même que ce sens-phénomène

12. Erwin Panofsky, L'œuvre d'art et ses significations, Gallimard, Paris, 1969. 
dont il prend le relais ne peut être atteint qu'à travers la correspondance forme/chose, le sens-signification, c'est-àdire la maîtrise du sens, n'est accessible qu'à travers des étapes de marquage, puis de déchiffrage des constituants iconiques et graphiques du tableau.

Alors que la saisie phénoménologique de la convention représentative est d'abord liée au procès perceptif, au niveau objectal, selon Panofsky, c'est comme si on substituait au référent réel (le représenté) le référent peint (le représentant), occultant définitivement la chose-forme au profit du signe (de représentation). Puis, le code iconographique fait intervenir la "compétence linguistique", encyclopédique, du regardeur. Selon Panofsky, on en appelle ainsi, au gré d'une métaphorisation visuelle, à une représentation dans laquelle un certain sens-chose s'est allié à un certain sens-signification de façon si indissociable que cette représentation est traditionnellement devenue le porteur de ce sens-signification. C'est l'organisation syntaxique de sa pluralité d'éléments constitutifs qui rendrait possible de voir et de décrire le tableau.

La description, structure analytique par excellence de la surface du tableau pour l'analyse panofskienne, appelle des prolongements en structure profonde sur le plan sémiotique. Dans la décomposition du tableau peint en figures et en espaces de la représentation, puis dans la déconstruction de ceux-ci en leurs éléments, la structure supportée n'est autre qu'un modèle programmatique/ reprogrammatique. Dans son ensemble, le dispositif descriptif est alors une systématicité analytique, une métacommunication théorique, qui implique toujours une articulation verticale à partir d'un niveau pré-conscient où le sens-phénomène s'in-forme dans le procès perceptif jusqu'aux signes de la manifestation visuelle. La description du contenu en histoire de l'art, notamment pour l'iconologie, suppose donc qu'entre le regardeur/descripteur et le tableau s'institutionnalise déjà un langage, une valeur 
d'image normée, codée, produite par les instances convoquées et évoquées plus haut, ce qui instaure entre le tableau et sa description une médiation sémiotisable. Tout est là : la figure formée, élément paramimétique du tableau, et les stratégies graphiques-perspectives de la représentation, qui ne peuvent imposer leur domination que parce que le regardeur a été disposé, instruit, programmé à ne voir qu'eux et à tout leur rapporter ${ }^{13}$.

\section{Construction d'un modèle}

Suivant une direction générale de recherche déjà esquissée par le Groupe Mu14, et au Québec par René Payant ${ }^{15}$, je vois dans ces instances de la spatialisation et de l'iconisation une structure préalable aux univers de l'énoncé et de l'énonciation, dont la dynamique rendrait compte d'une conception saussuro-hjelmslévienne du signe, où dualisme ( $\mathrm{Sa} / \mathrm{Sé}$, signe/objet) et intégration verticale définissent la relation des termes.

Pendant les années 70 , le Groupe Mu aura établi qu'une rhétorique de l'image fixe n'était possible qu'à la condition de distinguer deux signes : le signe proprement iconique, analogique, et le signe plastique. Pour le Groupe, c'étaient là les domaines de base qu'une sémiotique visuelle devait investir, séparément, puis dans leurs relations. Payant a utilisé cette méthode, conservant le modèle d'ensemble du Groupe Mu, tout en optant cependant pour le signe pictural révélé par la critique formaliste de l'art abstrait au lieu du signe plastique. Déjà on sent la néces-

13. Bernard Vouilloux, "La description du tableau", in Poétique, 73 , février 1988, p. 27-50.

14. Groupe $\mathrm{Mu}$, “conique et plastique : sur un fondement de la rhétorique visuelle", in Revue d'esthétique, 1-21, 1979, p. 173-192; “Plan d'une rhétorique de l'imagew, Kodikas-Code, 3, 1980, p. 249-268.

15. René Payant, Vedute. Pièces détachées sur l'art, éd. Trois, Laval, 1987, p. 57-58. 
sité de recourir à un modèle qui serait doté d'une hiérarchie verticale pour rendre compte des structures profondes du langage visuel. Au plan de la stratification du tableau, ce modèle engageait dorénavant le regardeur dans un mouvement de va-et-vient entre l'image peinte et la matière, donc dans l'exploration des passages du champ de l'iconique à celui du pictural (plastique) : le pictural étant le lieu de la manipulation et de l'organisation des lignes, couleurs, surfaces, et l'iconique, l'effet de sens produit par cette manipulation.

Ensuite, pour dresser le système complexe du tableau, Payant a introduit les distinctions de Hjelmslev entre substance et forme du contenu, déjà annoncées par Saussure en termes de strata à l'intérieur du signe : l'iconique étant la forme, le pictural, la substance. La construction de cette réalité formelle et substantielle lui permet de s'opposer, par exemple, à la scénographie avancée par Schefer ${ }^{16}$, laquelle abordait le tableau avec des "niveaux de lexies», la lecture iconique déterminant l'image de ses structures.

Cependant, si ce modèle postulait d'entrée de jeu l'existence d'une morphogénèse matérielle, autant le Groupe Mu que Payant auront inscrit le déplacement entre l'iconique et le plastique (pictural) dans un schéma de transition orienté, basé sur le processus d'iconisation, de figuration (donc, sur la forme). Là, tout écart par rapport au mode pressenti de reconnaissance mimétique se posait, vis-à-vis du tableau, comme un travail de dé-figuration, comme des procédés de dé-iconisation, ce qui aura réinstallé liillusion référentielle en tant que figure prédominante dans la démonstration. Autrement dit, bien qu'elle ressourçait alors la translation du modèle linguistique en art, cette modélisation s'est montrée exagérément centrée sur les

16. Jean-Louis Schefer, Scénographie d'un tableau, Seuil, Paris, 1968. 
rapports énonciatifs du lisible et du visible. C'est pourquoi, contrairement à cette démarche, malgré mon recours par commodité analytique à une systématicité similaire, soit le modèle de Hjemslev, il ne s'agit plus de lire des contenus visuels selon le mode de décodage de la chaîne parlée. Je veux plutôt, en première instance, délimiter une procédure méta-théorique, para-linguistique, qui me permette de prioriser les qualités ou valeurs propres du tableau.

Substituant le perspectif au plastique (au pictural) dans le modèle, je souligne que le système perspectiviste délimite le perçu et le nommé dans le champ visuel, positionne, mais aussi intègre l'objet dans un continu significatif, en ce qu'il démontre critiquement comment l'image peinte et son référent dans le monde réel entretiennent effectivement un rapport, la première étant un mode de connaissance du second. Ce rapport qui est ainsi établi, et qui tend généralement à l'iconisme comme critère d'interprétation, m'apparaît recouvrir, sémiotiquement parlant, un espace de profondeur simulé par le système perspectiviste de la peinture. Cet espace de la perspective construit l'apparence mimétique, ainsi que l'artifice d'une profondeur de l'image peinte qui confond l'horizontalité de la surface, c'est-à-dire que la perspective permet la transcription d'une représentation imagière en trois dimensions sur la bidimensionnalité de la surface du support. C'est ainsi que la construction structurelle d'une double spatialité présiderait d'entrée de jeu à la considération du contenu de l'image picturale.

Les greimassiens parlent de l'espace perspectiviste comme d'une isotopie dominante, au sens où elle crée l'illusion selon une cohérence sémantique de l'énoncé, celle du discours visuel produit par ses valeurs prescriptives. Floch et Thürlemann y voient une structure superficielle de l'énoncé puisqu'ils réservent le niveau le plus profond à la mise en discours, c'est-à-dire à ce qui relève de la rhétorique qui affecterait le procès de l'énonciation 
visuelle à la base ${ }^{17}$. II m'apparaît plutôt que l'édification graphique de l'image-plan consiste en un acte d'inférence de la substance à la forme. Cet acte reposant sur des codes perspectifs et iconiques qui assertent les constituants visuels et plastiques de l'œuvre. En termes hjelmsléviens, dans les profondeurs du tableau, au plan intégral de la matière du contenu (MC), une réalité formelle (iconique) serait constituée (formée) par une réalité substantielle (perspective) comme structure sémiotique sous-jacente à l'univers du visible.

À l'encontre des théories basées sur la forme, je propose une ordination du contenu qui est basée sur la substance, et qui me permet de réévaluer les rapports des éléments constitutifs, de comprendre autrement le statut d'indépendance relative ou de dépendance réciproque de ces instances structurelles de l'image peinte. Le schéma qui suit résume cette conception fondée sur l'idée de subordination hiérarchique :

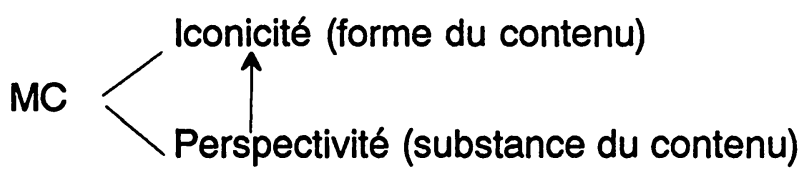

Admettre ces distinctions implique que le travail au premier niveau de composition de l'image peinte, celui des réseaux graphiques et leur rapport à la surface, lequel produit un effet spatialisant répercuté jusqu'au niveau de la

17. Jean-Marie Floch, Petites mythologies de l'œil et de l'esprit. Pour une sémiotique plastique, éd. Hadès-Benjamin, Paris, 1985; Félix Thürlemann, Paul Kee, Analyse sémiotique de trois peintures, L'Âge d'Homme, Lausanne, 1982; "Fonctions cognitives d'une figure de perspective picturale», in Le Bulletin du Groupe de Recherche Sémio-linguistique, 15, septembre 1980, p. 37-47. 
forme, passe par différents systèmes perspectifs, du lointain au proxémique, qui (dé)voilent littéralement les mécanismes de la figuration. Ils rendent perceptibles les diverses formes d'iconicité de l'image : images figuratives, semi-figuratives, semi-abstraites et abstraites ${ }^{18}$. Sousjacente à l'iconicité, il existerait donc une typologie des perspectives comme mode de spatialité et d'organisation du visible, d'où un espace de circulation de la substance à la forme, une hiérarchie des relations, un langage intersémiotique du visuel. À partir de ce nœud structural, s'élaborerait une mise en réseau de traits caractéristiques, descriptifs et analytiques, qui prend ou fait sens, qui marque l'intervention de la spécificité visuelle : à savoir le fait d'être une pratique dont la matière visuelle et plastique est homogène, proprement spatiale, et non un mélange de lisible et de visible dans la foulée d'une rhétorique barthésienne de l'image ${ }^{19}$.

\section{Bref...}

La contribution essentielle de ce modèle sémiotique est d'insister qu'en même temps la représentation figurale et le dispositif optique qui la fondent, déterminent le primat phénoménologique de la figure, mais aussi d'avoir télescopé les équivalences entre le sens-chose et le senssignification en fonction d'une scientificité qui en instaure structuralement les rapports, les correspondances. On rejoint ainsi la problématique de la lecture descriptive du contenu de l'œuvre d'art inspirée de Panofsky, et particulière à la sémiotique visuelle.

18. Marie Carani, "Sémiotique de l'abstraction picturalem, Semiotica, 67, 1-2, p. 1-39.

19. Marie Carani, Études sémiotiques sur la perspective, Cahiers du GRESAC $n^{\circ} 1$, département d'histoire, Université Laval, 1989. 
Dans ce contexte, la stratégie théorique dont je viens de conjuguer quelques flexions comme effectuation du modèle, s'inscrit dans le sens d'une sémiotisation faisant passer l'objet d'analyse du syntaxique au syntagmatique, au textuel comme tel. Modélisation visuelle sous forme de modus inextricable au cœur de laquelle chaque œuvre picturale se cristallise dans le jeu complexe des intervalles, des graphismes et des figures, sorte de lieu nodal, espace élargi de circulation, nouvelle synthèse où gravitent les productions figuratives, néo-figuratives ou abstraites dont la discipline de l'histoire de l'art prétend parler, et dont la sémiotique visuelle permet le déplacement du signe comme tel à la (re)présentation méta-langagière de la représentation.

L'idée directrice de cette "sémiotique perspectiviste" est ainsi de déborder le mode iconique par l'adjuvant spatialisant qui le détermine, le saisit, le fouille. Le travail de déplacement qu'opère cette approche aboutit à établir des continuités, donc un continu, entre des éléments visuels et plastiques demeurés jusque là le plus souvent écartés, séparés. Maintenant, ceux-ci se rapprochent, s'interpénètrent même. Et, c'est dans l'optique de cette notion du continu qu'on a suggéré ici d'approcher l'objet pictural par les instruments modélisants de la sémiotique hjelmslévienne, relayés par ceux que développe la sémiotique de la perspective.

Marie Carani Université Laval 


\section{Bibliographie pertinente}

Carani, Marie, Études sémiotiques sur la perspective, Cahiers du GRESAC, no 1, département d'histoire, Université Laval, 1989, 214 pages.

Damisch, Hubert, L'origine de la perspective, Flammarion, Paris, 1987, 408 pages.

Eco, Umberto, A Theory of Semiotics, Indiana University Press, Bloomington, Ind., 1983, 354 pages.

Groupe Mu, Rhétorique générale, Éd. Complexe, Bruxelles, 1977, 296 pages.

Lindekens, René, Essai de sémiotique visuelle, Klincksieck, Paris, 1976, 200 pages.

Panofsky, Erwin, La perspective comme forme symbolique, Minuit, Paris, 1976, 273 pages.

Panofsky, Erwin, L'œurre d'art et ses significations, Gallimard, Paris, 1969, 283 pages.

Saint-Martin, Fernande, Sémiologie du langage visuel, PUQ, Sillery, 1987, 307 pages.

Schapiro, Meyer, Words and Pictures, Mouton, The Hague-Paris, 1973, 108 pages. 